\title{
SPECTRAL PROPERTIES OF ORTHOGONAL POLYNOMIALS ON UNBOUNDED SETS ${ }^{1}$
}

BY

\author{
T. S. CHIHARA
}

\begin{abstract}
We consider orthogonal polynomials when the three term recurrence formula for the monic polynomials has unbounded coefficients. We obtain information relative to three questions: Under what conditions on the coefficients will the derived set of the spectrum have a finite infimum $\sigma$ ? If $\sigma$ is finite, when will there be at most finitely many spectral points smaller than $\sigma$; and when will the distribution function be continuous at $\sigma$ ?
\end{abstract}

0. Introduction. Let $\mathbf{P}=\left\{P_{n}(x)\right\}_{n=0}^{\infty}$ be a sequence of orthogonal polynomials defined by

$$
\begin{gathered}
P_{n}(x)=\left(x-c_{n}\right) P_{n-1}(x)-\lambda_{n} P_{n-2}(x), \quad n \geqslant 1, \\
P_{-1}(x)=0, \quad P_{0}(x)=1, \quad c_{n} \text { real }, \lambda_{n}>0 .
\end{gathered}
$$

In $1898, \mathrm{O}$. Blumenthal [4] proved that if

$$
\lim _{n \rightarrow \infty} c_{n}=c, \quad \lim _{n \rightarrow \infty} \lambda_{n}=\lambda
$$

are finite, then the zeros of the $P_{n}(x)$ are dense in $(\sigma, \tau)$,

$$
\sigma=c-2 \sqrt{\lambda}, \quad \tau=c+2 \sqrt{\lambda} .
$$

In addition, $\sigma$ and $\tau$ are the smallest and largest limit points of $\delta(\psi)$, the spectrum of "the" distribution function with respect to which the $P_{n}(x)$ are orthogonal. In recent years, there has been a great deal of interest in questions involving the spectral properties of $\psi$ when the conditions of Blumenthal's theorem are satisfied. (For references, see [9]. See also the recent papers by Geronimo and Case [11] and Nevai $[13,14]$.) In particular, Nevai [12] has proved that if $\Sigma_{n=1}^{\infty}\left\{\left|c_{n}-c\right|+\left|\lambda_{n}-\lambda\right|\right\}<\infty$ then $\psi$ is absolutely continuous on $(\sigma, \tau), \psi^{\prime}$ is positive and continuous on $(\sigma, \tau)$, and $\psi$ has at most countably many spectral points on the complement of $(\sigma, \tau)$. (The latter assertion is implied by Blumenthal's theorem.)

Among the questions addressed in these studies are: Under what additional hypotheses on $c_{n}$ and $\lambda_{n}$ will $\delta(\psi) \cap(\sigma, \tau)^{c}$ be finite? Will $\psi$ be continuous at $\sigma$ and $\tau$ ? It seems natural to extend such questions to analogous situations when the limits $(0.2)$ are infinite. For the questions to be meaningful, we have to generalize the definitions of $\sigma$ and $\tau$.

Received by the editors January 6, 1981; presented to the Society, in San Francisco, California, January 7, 1981.

1980 Mathematics Subject Classification. Primary 42C05; Secondary 33A65.

${ }^{1}$ Research supported by National Science Foundation grant No. MCS 80-01792. 
Let $x_{n i}=x_{n i}(\mathbf{P})$ denote the zeros of $P_{n}(x)$ :

$$
x_{n 1}<x_{n 2}<\cdots<x_{n n},
$$

and set

$$
\begin{gathered}
\xi_{i}=\xi_{i}(\mathbf{P})=\lim _{n \rightarrow \infty} x_{n i}, \quad \eta_{j}=\lim _{n \rightarrow \infty} x_{n, n-j+1}, \\
\boldsymbol{\sigma}=\boldsymbol{\sigma}(\mathbf{P})=\lim _{n \rightarrow \infty} \xi_{i}, \quad \tau=\lim _{j \rightarrow \infty} \eta_{j} .
\end{gathered}
$$

When (0.2) is satisfied, these agree with (0.3). If $c_{n} \rightarrow \infty(n \rightarrow \infty)$, then $\tau=\infty[8, \mathrm{p}$. 113].

The only analog of Blumenthal's theorem for unbounded coefficients that we know of is the following $[8$, p. 125]: Let

$$
\lim _{n \rightarrow \infty} c_{n}=c, \quad \lim _{n \rightarrow \infty} \frac{\lambda_{n+1}}{c_{n} c_{n+1}}=\frac{1}{4} .
$$

If $\sigma$ is finite, the zeros of the $P_{n}(x)$ are dense in $(\sigma, \infty)$.

Unfortunately, we do not have an analog of Nevai's theorem for this case. In fact, although the definition of $\sigma$ makes it clear that, when it is finite, $\sigma$ is the least limit point of $\mathcal{S}(\psi)$, we do not even know if $\mathcal{S}(\psi) \cap[\sigma, \infty)=[\sigma, \infty)$. (When $\sigma$ is finite, the Hamburger moment problem is determined [7] so $\psi$ is uniquely determined up to an equivalence class and $\delta(\psi)$ is unique.) Nevertheless, we believe information of sufficient interest can be found to justify a detailed study.

We will address three questions:

(i) Under what additional conditions on $c_{n}$ and $\lambda_{n}$ will $\sigma$ be finite?

(ii) If $|\sigma|<\infty$, when will $\psi$ have at most finitely many spectral points smaller than $\sigma$ ?

(iii) If $|\sigma|<\infty$, when will $\psi$ be continuous at $\sigma$ ?

Although these questions constitute the initial motivation for this study, our analysis will lead naturally to the discovery of new theorems yielding the conclusions $\sigma=-\infty$ and $\sigma=\infty$. Our findings will also enable us to make a complete analysis (relative to these three questions) of all cases where $c_{n}$ and $\lambda_{n}$ are polynomials in $n$.

1. Preliminaries. If $\mathbf{a}=\left\{a_{n}\right\}$ is a numerical sequence, we write

$$
a_{n}^{(N)}=a_{n+N}, \quad \mathbf{a}^{(N)}=\left\{a_{n}^{(N)}\right\} .
$$

With reference to $(0.1)$, we let

$$
\begin{gathered}
\alpha_{n}(x)=\frac{\lambda_{n+1}}{\left(c_{n}-x\right)\left(c_{n+1}-x\right)}, \quad \boldsymbol{\alpha}(x)=\left\{\alpha_{n}(x)\right\}, \\
\alpha_{n}(0)=\alpha_{n}, \quad \alpha=\left\{\alpha_{n}\right\} .
\end{gathered}
$$

We denote by $\mathbf{P}^{(N)}=\left\{P_{n}^{(N)}(x)\right\}$ the sequence of numerator polynomials of order $N$ :

$$
P_{n}^{(N)}(x)=\left(x-c_{n}^{(N)}\right) P_{n-1}^{(N)}(x)-\lambda_{n}^{(N)} P_{n-2}^{(N)}(x) .
$$

(No confusion should arise between the meaning of $\alpha_{n}^{(N)}(x)=\alpha_{n+N}(x)$ and $P_{n}^{(N)}(x)$ which will never denote $P_{n+N}(x)$.) 
We recall that $P_{n+N}(x)$ has at most $N$ zeros smaller than the least zero of $P_{n}^{(N)}(x)$. It follows that

$$
\xi_{1}\left(\mathbf{P}^{(N)}\right) \leqslant \xi_{N+1}(\mathbf{P}) .
$$

As in our previous studies of (0.1), we will make extensive use of Wall's theory of chain sequences [17] (see also [8]). We write

$\mathbf{a} \in \mathcal{C}$ if $a_{n}>0(n \geqslant 1)$ and $\mathbf{a}$ is a chain sequence;

$\mathbf{a} \in \mathcal{E}$ if there exists $N$ such that $\mathbf{a}^{(N)} \in \mathcal{C}$.

In the latter case, we say $\mathbf{a}$ is an eventual chain sequence. We deal exclusively with positive chain sequences and the adjective will be omitted.

Our analysis will be based on the following fundamental theorem.

THEOREM 1.1. Let $c_{n} \rightarrow \infty$.

(i) If $\boldsymbol{\alpha}(x) \in \mathcal{E}$, then $\sigma \geqslant x$.

(ii) If $\boldsymbol{\alpha}(x) \notin \mathcal{E}$, then $\sigma \leqslant x$.

(iii) If $\sigma$ is finite, a necessary and sufficient condition for $\delta(\psi) \cap(-\infty, \sigma)$ to be a finite set is $\boldsymbol{\alpha}(\sigma) \in \mathcal{E}$.

Proof. The first two assertions are restatements of an earlier theorem [8, p. 114] which also gives the necessary condition in (iii). To prove the sufficiency in (iii), let $\boldsymbol{\alpha}(\sigma) \in \mathcal{E}$. Then there is an $N$ such that $c_{n}^{(N)}>\sigma(n \geqslant 1)$ and $\boldsymbol{\alpha}^{(N)}(\sigma) \in \mathcal{C}$. Thus, the spectral interval (i.e., the "true interval of orthogonality") for $\mathbf{P}^{(N)}$ is a subset of $[\sigma, \infty)$. It follows from $(1.4)$ that $\delta(\psi)$ has at most $N$ points smaller than $\sigma$.

Theorem 1.1 reduces much of our work to finding conditions on $(0.1)$ for $\alpha(x)$ to be in $\mathscr{E}$ or in $\mathcal{E}^{c}$. Determination of such conditions will frequently be made with the aid of Wall's comparison tests [17] (see also [8, pp. 95, 97]).

THEOREM 1.2. Let $\left\{b_{n}\right\}$ be a chain sequence with parameter sequence $\left\{g_{n}\right\}$. If $0<a_{n} \leqslant b_{n}, n \geqslant 1$, then $\left\{a_{n}\right\}$ is also a chain sequence and its minimal and maximal parameters, $m_{n}$ and $M_{n}$, satisfy

$$
m_{n} \leqslant g_{n} \leqslant M_{n}, \quad n \geqslant 0 .
$$

Turning now to $(0.1)$, we can write $\alpha_{n}(x)=\frac{1}{4}+\varepsilon_{n}(x)$ where

$$
\begin{aligned}
\varepsilon_{n}(x)= & \frac{x}{4}\left[\frac{1}{c_{n}-x}+\frac{1}{c_{n+1}-x}+\frac{1}{\left(c_{n}-x\right)\left(c_{n+1}-x\right)}\right] \\
& +\frac{\varepsilon_{n} c_{n} c_{n+1}}{\left(c_{n}-x\right)\left(c_{n+1}-x\right)} \quad\left(\varepsilon_{n}=\varepsilon_{n}(0)=\alpha_{n}-\frac{1}{4}\right) .
\end{aligned}
$$

Let $\boldsymbol{\beta}=\left\{\beta_{n}\right\}$ be any positive sequence. Elementary calculations show that $\alpha_{n}(x) \geqslant \beta_{n}$ if and only if $A_{n}(\boldsymbol{\beta}) \leqslant x \leqslant B_{n}(\boldsymbol{\beta})$ where

$$
2 A_{n}(\boldsymbol{\beta})=c_{n}+c_{n+1}-\left[\left(c_{n}+c_{n+1}\right)^{2}+4 \beta_{n}^{-1}\left(\alpha_{n}-\beta_{n}\right) c_{n} c_{n+1}\right]^{1 / 2}
$$

and $B_{n}(\boldsymbol{\beta})$ is the conjugate surd. $A_{n}(\boldsymbol{\beta})$ and $B_{n}(\boldsymbol{\beta})$ are real if $c_{n}>0, c_{n+1}>0$. Thus, if $c_{n} \rightarrow \infty$, then $B_{n}(\boldsymbol{\beta}) \rightarrow \infty$.

We can rewrite (1.6) as

$$
A_{n}(\boldsymbol{\beta})=\left(\beta_{n}-\alpha_{n}\right) R_{n}(\boldsymbol{\beta})
$$


We have previously given sufficient conditions for

$$
a_{n}=\frac{1}{4}+\frac{1+e_{n}}{16 n(n+1)}, \quad n \geqslant 1,
$$

to yield an eventual chain sequence. We now obtain a necessary condition which will verify our conjecture about (2.4). For convenience of reference, we include our earlier results in the statement of the theorem.

THEOREM 2.2. Let $\mathbf{a}=\left\{a_{n}\right\}$ have the form (2.5).

(i) If either $e_{n}=O\left(n^{-1}\right)$ or $\sum e_{n}$ converges, then $\mathbf{a} \in \mathcal{E}$.

(ii) If $e_{n} \geqslant 0$ ( for all $n$ sufficiently large) and $\sum e_{n} / n=\infty$, then a $\notin \mathcal{E}$.

Proof. (i) was proved in [9, p. 362]. For the proof of (ii), consider the chain sequence $\mathbf{b}(s)$ :

$$
b_{n}(s)=\frac{1}{4}+\frac{s(1-s)}{4 n(n+1)}, \quad 0<s \leqslant \frac{1}{2},
$$

which has the parameters $g_{n}(s)=(n+s) /(2 n+2), n \geqslant 0$. Wall's criterion shows that $\mathbf{g}\left(\frac{1}{2}\right)$ is a maximal parameter sequence while $\mathbf{g}(s)$ is not maximal if $s<\frac{1}{2}$.

Now assume $\mathbf{a}^{(N)} \in \mathcal{C}$ and let $\mathbf{h}=\left\{h_{n}\right\}$ be any parameter sequence for $\mathbf{a}^{(N)}$. Let $\mu_{n}(s)$ denote the minimal parameters for $\mathbf{b}^{(N)}(s)$. By Theorem 1.2, we have

$$
\mu_{n}(s) \leqslant h_{n} \leqslant g_{n}^{(N)}\left(\frac{1}{2}\right), \quad 0<s<\frac{1}{2} .
$$

Next let

$$
t_{n}=2(n+N+1) h_{n}-(n+N)
$$

so that $h_{n}=\left(n+N+t_{n}\right) /[2(n+N+1)]$. We can then write

$$
a_{n}^{(N)}=\frac{1}{4}+\frac{\left(1-t_{n-1}\right) t_{n}+(n+N)\left(t_{n}-t_{n-1}\right)}{4(n+N)(n+N+1)} .
$$

Thus

$$
e_{n}^{(N)}=4 v_{n}+4(n+N)\left(t_{n}-t_{n-1}\right)
$$

where $w_{n} \equiv\left(1-t_{n-1}\right) t_{n}=\frac{1}{4}+v_{n}$. Using (2.6) and (2.7), we find $t_{n}<\frac{1}{2}$ and

$$
t_{n} \geqslant 2(n+N+1)\left[g_{n}^{(N)}(s)-D_{n}(s)\right]-(n+N)=s-2(n+N+1) D_{n}(s)
$$

where $D_{n}(s)=g_{n}^{(N)}(s)-\mu_{n}(s)$. But by Lemma 2.2, $n D_{n}(s) \rightarrow 0\left(0<s<\frac{1}{2}\right)$; hence $t_{n} \rightarrow \frac{1}{2}(n \rightarrow \infty)$.

It now follows that, for some $k, \mathbf{w}^{(k)} \in \mathcal{C}$ and by Lemma $2.1, \sum v_{n}^{(k)} \leqslant \frac{1}{4}$. Thus referring to (2.8),

$$
\sum_{n=1}^{\infty} \frac{e_{n}^{(N)}}{n+N} \leqslant 4 \sum_{n=1}^{\infty} \frac{v_{n}}{n+N}+4\left(\frac{1}{2}-t_{0}\right)<\infty .
$$

REMARK. It would be useful to improve the inequalities in Theorem 2.1, especially the left side of (ii). The possibility of decided improvement is indicated by the following 
THEOREM 2.3. If $\mathbf{a} \in \mathcal{C}$ and $M_{0}(\mathbf{a})>0$, then

$$
\sum_{n=1}^{\infty} \frac{\Delta_{n}(\mathbf{a})}{m_{n}(\mathbf{a})\left[1-M_{n}(\mathbf{a})\right]}=\infty \text {. }
$$

In particular, if $a_{n} \geqslant r>0$, then $\Sigma \Delta_{n}(\mathbf{a})=\infty$.

Proof. Let

$$
A_{n}=\prod_{k=1}^{n} \frac{m_{k}}{1-m_{k}}, \quad B_{n}=\prod_{k=1}^{n} \frac{M_{k}}{1-M_{k}} .
$$

Again using Wall's criterion, we have $\sum A_{n}<\infty$ and $\sum B_{n}=\infty$. We also have the identity

$$
\frac{B_{n}}{A_{n}}=\prod_{k=1}^{n}\left[1+\frac{M_{k}-m_{k}}{m_{k}\left(1-M_{k}\right)}\right] .
$$

Since $B_{n} / A_{n} \rightarrow \infty$, we obtain (2.9). If also $a_{n} \geqslant r>0$, then all parameters are bounded away from both 0 and 1 so $\Sigma \Delta_{n}=\infty$.

3. Conditions for $|\sigma|=\infty$. Before taking up the case $|\sigma|<\infty$, we consider the opposite situation. We recall that if $c_{n} \rightarrow \infty$, then

$$
\limsup _{n \rightarrow \infty} \alpha_{n}<\frac{1}{4} \Rightarrow \sigma=\infty, \quad \liminf _{n \rightarrow \infty} \alpha_{n}>\frac{1}{4} \Rightarrow \sigma=-\infty
$$

[8, pp. 109, 119]. We now show that even if $c_{n} \rightarrow \infty$ and $\alpha_{n} \rightarrow \frac{1}{4}$, no additional condition on $\left\{c_{n}\right\}$ alone is sufficient to conclude $|\sigma|<\infty$.

THEOREM 3.1. Given any $c_{n}$ with $c_{n} \rightarrow \infty$,

(i) there exist $\lambda_{n}$ such that $\alpha_{n} \rightarrow \frac{1}{4}$ and $\sigma=\infty$;

(ii) there exist $\lambda_{n}$ such that $\alpha_{n} \rightarrow \frac{1}{4}$ and $\sigma=-\infty$.

Proof. (i) Choose any $\varepsilon_{n}<0$ such that $\varepsilon_{n} \rightarrow 0$ and

$$
\lim _{n \rightarrow \infty}\left|\varepsilon_{n}\right| \min \left(c_{n}, c_{n+1}\right)=\infty \text {. }
$$

Let $\lambda_{n+1}=\alpha_{n} c_{n} c_{n+1}$, where $\alpha_{n}=\frac{1}{4}+\varepsilon_{n}$. Then taking $\beta_{n}=\frac{1}{4}$, we have from (1.8) that $A_{n}(\boldsymbol{\beta}) \rightarrow \infty$. By Theorem 1.3, $\sigma=\infty$.

(ii) Take $\gamma_{n} \geqslant \frac{1}{4}, \gamma_{n} \rightarrow \frac{1}{4}$ such that $\Sigma\left(\gamma_{n}-\frac{1}{4}\right)=\infty$. Then $\gamma \notin \mathcal{E}[8$, p. 99].

Now choose $\lambda_{n}$ so that

$$
\alpha_{n}=\gamma_{n}+a_{n}^{-1}, \quad a_{n}=\left[\min \left(c_{n}, c_{n+1}\right)\right]^{1 / 2} .
$$

Then, again by (1.8), $A_{n}(\gamma)=-a_{n}^{-1} R_{n}(\gamma) \rightarrow-\infty$ so $\sigma=-\infty$.

By contrast, we note that if $c_{n}$ grows too rapidly and $\alpha_{n} \rightarrow \frac{1}{4}, \sigma$ cannot be finite.

THEOREM 3.2. Let $c_{n} \rightarrow \infty, \alpha_{n} \rightarrow \frac{1}{4}$. If $\liminf _{n \rightarrow \infty} c_{n}^{1 / n}>1$ then $|\sigma|=\infty$. Furthermore, if $\sigma=\infty$, the associated Hamburger moment problem is indeterminate.

Proof. Suppose $\sigma>-\infty$. Then there exist $t$ and $N$ such that

$$
c_{n}^{(N)}>t, \quad \boldsymbol{\alpha}^{(N)}(t) \in \mathcal{C} .
$$


We then consider in place of $\left\{P_{n}(x)\right\}$ the numerator polynomials of order $N(1.3)$ with a translation in $x$ :

$$
Q_{n}(x)=P_{n}^{(N)}(x-t) .
$$

Then for $\mathbf{Q}=\left\{Q_{n}(x)\right\},(3.2)$ tells us that the spectral interval $\left[\xi_{1}(\mathbf{Q}), \infty\right)$ is a subset of $[0, \infty)$.

Also, corresponding to $\mathbf{Q}$ we have

$$
\lim _{n \rightarrow \infty} \alpha_{n}^{(N)}(x-t)=\frac{1}{4}, \quad \liminf _{n \rightarrow \infty}\left(c_{n}-t\right)^{1 / n}>1 .
$$

This shows that the Hamburger moment problem for $\mathbf{Q}$ is indeterminate $[\mathbf{6}$, Theorem 4.3]. According to Sherman [15, Theorem II], the moment problem for $P$ is then also indeterminate. (Note that determinacy of a Hamburger moment problem associated with (0.1) is invariant under translation.) But if the Hamburger moment problem is indeterminate when $\xi_{1}(\mathbf{P})>-\infty$ then $\sigma(\mathbf{P})=\infty$ [7, Theorem 5].

Theorem 3.2 suggests that for orthogonal $q$-polynomials with unbounded coefficients in (0.1), we should "normally" expect $|\sigma|=\infty$. That is, if the coefficients are reasonably well behaved, we would expect $c_{n}$ to behave like $p^{-n}$ for some $0<p<1$, and $\alpha_{n}$ to converge. If $\sigma=\infty$, we have a discrete spectrum so it is tempting to speculate there will be a discrete spectrum if $\sigma=-\infty$ also. Unfortunately nothing appears to be known about this situation (except in the symmetric case $c_{n}=0$ ).

An explicit example of $\sigma=\infty$ under the conditions of Theorem 3.2 is the special Al-Salam and Carlitz $q$-polynomial set $\left\{V_{n}^{(1)}(x)\right\}$ [2] for which $c_{n+1}=2 q^{-n}, \lambda_{n+1}=$ $q^{1-2 n}\left(1-q^{n}\right)$.The artificial example

$$
c_{n}=q^{-n} \quad(0<q<1), \quad \alpha_{n}=\frac{1}{4}+\frac{a}{16 n^{2}} \quad(a>1),
$$

shows that $\sigma=-\infty$ is possible (see Theorem 3.5). We know of no explicitly "known" examples of this case $\sigma=-\infty, \alpha_{n} \rightarrow \frac{1}{4}$. Indeed, the only examples we are aware of (excluding symmetric cases) in which $\sigma=-\tau=-\infty$ are the Meixner polynomials of the second kind and their generalizations by Pollaczek (see [8, pp. $179,186])$. For these, however, $\alpha_{n} \rightarrow L>\frac{1}{4}$.

In contrast with the above, there are many important examples studied in the literature in which $\sigma=\infty$ (Charlier, Meixner (first kind), Stieltjes-Wigert, etc.). However, except for the Al-Salam and Carlitz example just cited, these all have $\alpha_{n} \rightarrow L<\frac{1}{4}$. Despite this dearth of examples (or, perhaps, because of it), we next obtain conditions sufficient for $\sigma=\infty$ when $\alpha_{n}$ is allowed to converge to $\frac{1}{4}$.

Lemma 3.1. Let $\mathbf{Q}=\left\{Q_{n}(x)\right\}$ satisfy

$$
\begin{gathered}
Q_{n}(x)=\left(x-d_{n}\right) Q_{n-1}(x)-v_{n} Q_{n-2}(x), \quad n \geqslant 1, \\
Q_{-1}(x)=0, \quad Q_{0}(x)=1, \quad d_{n} \text { real }, \nu_{n}>0 .
\end{gathered}
$$

If $d_{n} \rightarrow \infty$ and for all $n \geqslant N$

$$
c_{n} \geqslant d_{n}, \quad \alpha_{n} \leqslant \beta_{n} \equiv v_{n+1} /\left(d_{n} d_{n+1}\right),
$$

then $\sigma(\mathbf{P}) \geqslant \sigma(\mathbf{Q})$. 
Proof. For real $x$ and all sufficiently large $n$, we have $d_{n}>x$ and

$$
\alpha_{n}(x) \equiv \alpha_{n} \frac{c_{n} c_{n+1}}{\left(c_{n}-x\right)\left(c_{n+1}-x\right)} \leqslant \beta_{n} \frac{d_{n} d_{n+1}}{\left(d_{n}-x\right)\left(d_{n+1}-x\right)} \equiv \beta_{n}(x) .
$$

Using Theorems 1.1 and 1.2, we have

$$
x<\boldsymbol{\sigma}(\mathbf{Q}) \Rightarrow \boldsymbol{\beta}(x) \in \mathcal{E} \Rightarrow \boldsymbol{\alpha}(x) \in \mathcal{E} \Rightarrow x \leqslant \boldsymbol{\sigma}(\mathbf{P}) .
$$

THEOREM 3.3. Let $c_{n}^{-1}=o\left(n^{-4}\right)$. If there exists a $\gamma \in \mathcal{E}$ such that $\gamma_{n} \geqslant \frac{1}{4}, \alpha_{n} \leqslant \gamma_{n}$, $n \geqslant N$, then $\sigma=\infty$.

Proof. Because of Lemma 3.1, it suffices to take $\alpha_{n}=\gamma_{n}$. For sufficiently large $N$, $\boldsymbol{\alpha}^{(N)} \in \mathcal{C}$ and $\boldsymbol{\alpha}_{n}^{(N)} \geqslant \frac{1}{4}$. Let $m_{n}=m_{n}\left(\boldsymbol{\alpha}^{(N)}\right)$ and $M_{n}=M_{n}\left(\boldsymbol{\alpha}^{(N)}\right)$ denote the minimal and maximal parameters of $\boldsymbol{\alpha}^{(N)}$. We can assume $M_{0}>0$ (see (2.2)).

Now choose $\boldsymbol{\beta}=\left\{\beta_{n}\right\}$ so that

$$
\beta_{n}^{(N)}=\left(1-g_{n-1}\right) g_{n}, \quad g_{n}=\frac{1}{2}\left[M_{n}+m_{n}\right] .
$$

Then $\boldsymbol{\beta}^{(N)} \in \mathcal{C}$ and, by $(2.3)$ and Theorem $2.1, \beta_{n}^{(N)}-\alpha_{n}^{(N)} \geqslant M_{0}^{2}[2 n(n+1)]^{-2}$. Referring again to (1.8) and Theorem 1.3, we conclude $\sigma=\infty$.

Theorems 4.2 and 5.2 which follow suggest that the condition $c_{n}=o\left(n^{-4}\right)$ in Theorem 3.3 could stand some weakening. This is also suggested by the remark preceding Theorem 2.3. We can conclude $\sigma=\infty$ under less severe growth conditions on $c_{n}$ if we are more specific about $\alpha_{n}$.

THEOREM 3.4. The following are sufficient for $\sigma=\infty$.

(i) $c_{n}^{-1}=o\left(n^{-2}\right), \alpha_{n}^{(N)} \leqslant \frac{1}{4}+s /\left[16 n^{2}\right](s<1)$.

(ii) $\sum\left(n / c_{n}\right)<\infty, \sum n\left|\alpha_{n}-\frac{1}{4}\right|<\infty$.

Proof. In (i), take

$$
\beta_{n}^{(N)}=\frac{1}{4}+(16 n(n+1))^{-1}
$$

and use (1.8) and Theorem 1.3.

In (ii), we can use (1.5) to show that

$$
n\left|\varepsilon_{n}(x)\right|=O\left(n / c_{n}\right)+O\left(n\left|\varepsilon_{n}\right|\right) \text {. }
$$

Thus $\sum n\left|\varepsilon_{n}(x)\right|<\infty$ so $\boldsymbol{\alpha}(x) \in \mathcal{E}[\mathbf{1 0}$, Lemma 2]; hence $\sigma=\infty$ by Theorem 1.1.

Finally, we observe that by methods analogous to the preceding we can easily obtain conditions for $\sigma=-\infty$ when $\alpha_{n} \rightarrow \frac{1}{4}$. The complete lack of explicit examples here inhibits us from pursuing this in great detail but we present one simple result.

THEOREM 3.5. Let $c_{n}^{-1}=o\left(n^{-2}\right), \alpha_{n}^{(N)} \geqslant \frac{1}{4}+t /\left[16 n^{2}\right], t>1$. Then $\sigma=-\infty$.

Proof. We need only take

$$
\beta_{n}^{(N)}=\frac{1}{4}+\frac{s}{16 n(n+1)}, \quad 1<s<t .
$$

Then $\boldsymbol{\beta} \notin \mathcal{E}$ so yet another reference to (1.8) and Theorem 1.3 yields the desired conclusion. 
where

$$
\begin{gathered}
R_{n}(\boldsymbol{\beta})=\frac{c_{n} c_{n+1}}{c_{n}+c_{n+1}} S_{n}(\boldsymbol{\beta}), \\
S_{n}(\boldsymbol{\beta})=\beta_{n}^{-1}\left\{1+\left[1+4\left(\frac{\alpha_{n}}{\beta_{n}}-1\right) \frac{c_{n} c_{n+1}}{\left(c_{n}+c_{n+1}\right)^{2}}\right]^{1 / 2}\right\}^{-1} .
\end{gathered}
$$

For the latter, we have $\frac{1}{2} \leqslant \beta_{n} S_{n}(\boldsymbol{\beta})<1$ so that (1.7) yields

$$
\frac{1}{2} \frac{\beta_{n}-\alpha_{n}}{\beta_{n}} \min \left(c_{n}, c_{n+1}\right) \leqslant A_{n}(\boldsymbol{\beta}) \leqslant \frac{\beta_{n}-\alpha_{n}}{\beta_{n}} \max \left(c_{n}, c_{n+1}\right) \text {. }
$$

Let

$$
A_{*}(\boldsymbol{\beta})=\liminf _{n \rightarrow \infty} A_{n}(\boldsymbol{\beta}), \quad A^{*}(\boldsymbol{\beta})=\limsup _{n \rightarrow \infty} A_{n}(\boldsymbol{\beta}) .
$$

Theorem 1.3. Let $c_{n} \rightarrow \infty$. Then $A_{*}(\boldsymbol{\beta}) \leqslant \sigma \leqslant A^{*}(\gamma)$ for all $\boldsymbol{\beta} \in \mathcal{E}$ and for all positive sequences $\gamma \notin \mathcal{E}$.

Proof. If $\boldsymbol{\beta} \in \mathcal{E}$ and $x<A_{*}(\boldsymbol{\beta}),(1.5)$ and Theorem 1.2 show $\boldsymbol{\alpha}(x) \in \mathcal{E}$. Theorem 1.1 then says $x \leqslant \sigma$.

Conversely, if $\gamma=\left\{\gamma_{n}\right\} \notin \mathcal{E}, \gamma_{n}>0$, then the same references show that $x>$ $A^{*}(\gamma) \Rightarrow x \geqslant \sigma$.

2. Chain sequences. We collect here a few new facts about chain sequences for which we will have use in the sequel. We begin with a simple identity.

If $\mathbf{a}=\left\{a_{n}\right\} \in \mathcal{C}$, we denote by $m_{n}(\mathbf{a})$ and $M_{n}(\mathbf{a})$ the $n$th minimal and maximal parameters of a. We then set

$$
\Delta_{n}(\mathbf{a})=M_{n}(\mathbf{a})-m_{n}(\mathbf{a}) .
$$

Since we deal exclusively with positive chain sequences, we have

$$
0<m_{n}(\mathbf{a}) \leqslant M_{n}(\mathbf{a})<1, \quad n \geqslant 1,
$$

and we note that $[8$, p. 92]

$$
\Delta_{n}(\mathbf{a})=0(n \geqslant 1) \Leftrightarrow \Delta_{0}(\mathbf{a})=0 .
$$

In particular, since [8, p. 94]

$$
M_{1}(\mathbf{a})=M_{0}\left(\mathbf{a}^{(1)}\right)
$$

we have $\Delta_{n}\left(\mathbf{a}^{(1)}\right)>0$ for $n \geqslant 0$.

If we set

$$
b_{n}=\left(1-g_{n-1}\right) g_{n}, \quad g_{n}=\frac{1}{2}\left[M_{n}(\mathbf{a})+m_{n}(\mathbf{a})\right],
$$

we have the identity

$$
b_{n}=a_{n}+\frac{1}{4} \Delta_{n-1}(\mathbf{a}) \Delta_{n}(\mathbf{a}) .
$$

Theorem 2.1. Let $\mathbf{a} \in \mathcal{C}$.

(i) If $a_{n} \leqslant \frac{1}{4}(n \geqslant 1)$, then $\Delta_{n} \geqslant[2(n+1)]^{-1}$.

(ii) If $a_{n} \geqslant \frac{1}{4}(n \geqslant 1)$, then $M_{0}(\mathbf{a})(n+1)^{-2} \leqslant \Delta_{n}(\mathbf{a}) \leqslant[2(n+1)]^{-1}$. 
Proof. The minimal and maximal parameters of the chain sequence $\left\{\frac{1}{4}\right\}$ are $n /(2 n+2)$ and $\frac{1}{2}$, respectively. Theorem 1.2 thus yields (i) and the right-hand inequality in (ii).

To complete the proof of (ii), we use the identity

$$
\Delta_{n}=\frac{M_{n}}{1-m_{n-1}} \Delta_{n-1}
$$

where $\Delta_{n}=\Delta_{n}(\mathbf{a})$, etc. Thus

$$
\Delta_{n}=\frac{M_{0} M_{n}}{1-m_{0}} \prod_{k=1}^{n-1} \frac{M_{k}}{1-m_{k}} \geqslant M_{0} M_{n} \prod_{k=1}^{n-1} \frac{m_{k}}{1-m_{k}} .
$$

Again using Theorem 1.2 and $\left\{\frac{1}{4}\right\}$, we obtain

$$
\Delta_{n} \geqslant M_{0} M_{n} \prod_{k=1}^{n-1} \frac{k}{k+2} \geqslant M_{0}(n+1)^{-2} .
$$

For use with Theorem 1.2, it is important to have examples of chain sequences that are as "large" as possible. We have previously observed that if we set

$$
a_{n}(s)=\frac{1}{4}+\frac{s}{16 n(n+1)}, \quad n \geqslant 1,
$$

then $\mathbf{a}(s) \in \mathcal{C}$ if and only if $-\frac{1}{2}<s \leqslant 1$. Further, $\mathbf{a}(s) \notin \mathcal{E}$ if $s>4$, and we conjectured that $\mathbf{a}(s) \notin \mathcal{E}$ if $s>1$ [9]. We will have need of the verification of this conjecture.

LeMma 2.1. Let $\mathbf{a} \in \mathcal{C}$. If a has a parameter sequence $\mathbf{g}$ with $g_{n} \leqslant \frac{1}{2}(n \geqslant 0)$ then $\sum_{n=1}^{\infty}\left(a_{n}-\frac{1}{4}\right) \leqslant \frac{1}{4}$.

Proof. Let $f_{n}=\frac{1}{2}-g_{n}$ so that $0 \leqslant f_{n} \leqslant \frac{1}{2}$. We have

$$
\begin{gathered}
a_{n}=\frac{1}{4}+\frac{1}{2}\left(f_{n-1}-f_{n}\right)-f_{n-1} f_{n}, \\
\sum_{k=1}^{n}\left(a_{k}-\frac{1}{4}\right)=\frac{1}{2}\left(f_{0}-f_{n}\right)-\sum_{k=1}^{n} f_{k-1} f_{k} \leqslant \frac{1}{2} f_{0} .
\end{gathered}
$$

Lemma 2.2. Let $\mathbf{a} \in \mathcal{C}$. If $a_{n} \geqslant \frac{1}{4}(n \geqslant 1)$ and if $\mathbf{g}$ is any nonmaximal parameter sequence for $\mathbf{a}$,

$$
\lim _{n \rightarrow \infty} n\left[g_{n}-m_{n}(\mathbf{a})\right]=0 .
$$

Proof. Let $D_{n}=g_{n}-m_{n}(\mathbf{a})$ so that

$$
D_{n}=\frac{g_{n}}{1-m_{n-1}} D_{n-1} \leqslant \frac{g_{n}}{1-g_{n-1}} D_{n-1} .
$$

Now $a_{n} \geqslant \frac{1}{4}$ implies $m_{n} \leqslant g_{n} \leqslant \frac{1}{2}$ so $D_{n} \leqslant D_{n-1}$. Also

$$
0 \leqslant D_{n} \leqslant \frac{g_{n}}{1-g_{0}} \prod_{k=1}^{n-1} \frac{g_{k}}{1-g_{k}} D_{0} .
$$

If $\mathbf{g}$ is not the maximal parameter sequence, Wall's criterion [8, p. 101] says that $\Sigma D_{n}$ converges. Since $\left\{D_{n}\right\}$ is monotonic, we conclude $n D_{n} \rightarrow 0$. 
4. $|\sigma|<\infty$; continuity at $\sigma$. Turning at last to the question of when $\sigma$ is finite, we first note that in this case the Hamburger moment problem is determined so the distribution function is substantially unique. That is, all equivalent distribution functions have the same spectrum so we will write $\psi \in \mathbb{Q}(t)$ if and only if $\sigma=t$ (finite) and $S(\psi) \cap(-\infty, t)$ is a finite set. Thus when $c_{n} \rightarrow \infty, \psi \in \mathbb{Q}(t)$ if and only if $x \leqslant t \Rightarrow \boldsymbol{\alpha}(x) \in \mathcal{E}$ and $x>t \Rightarrow \boldsymbol{\alpha}(x) \notin \mathcal{E}$.

We will find that, analogously with the situation that occurs when the coefficients are bounded (cf. [9]), the conditions

$$
c_{n}=O\left(n^{2}\right), \quad \alpha_{n}=\frac{1}{4}+O\left(n^{-2}\right)
$$

describe a borderline case.

Although a few of the following theorems remain true when $\left\{c_{n}\right\}$ converges to a finite limit, we will maintain without further mention the blanket hypothesis $c_{n} \rightarrow \infty$.

THEOREM 4.1. Let $\alpha_{n}=\frac{1}{4}+O\left(n^{-2}\right)$.

(i) If $c_{n}=O\left(n^{2}\right)$, then $\sigma$ is finite.

(ii) If $c_{n}=o\left(n^{2}\right)$, then $\sigma=0$.

Proof. Let

$$
\beta_{n}=\frac{1}{4}+\left(1+s_{n}\right) /\left[16 n^{2}\right], \quad s_{n} \rightarrow 0 .
$$

Using (1.8), we see that, in case (i), $A_{n}(\boldsymbol{\beta})$ is bounded while, in case (ii), $A_{n}(\boldsymbol{\beta}) \rightarrow 0$. But according to Theorem 2.2, we can choose $s_{n}$ so that either $\boldsymbol{\beta} \in \mathcal{E}$ (e.g. $s_{n} \leqslant 0$ ) or $\boldsymbol{\beta} \notin \&\left(\right.$ e.g. $\left.s_{n}=(\log n)^{-1}\right)$. Thus Theorem 1.3 shows that $\sigma$ is finite in (i) and $\sigma=0$ in (ii).

Theorem 4.1(ii) is sufficiently general to apply to the Laguerre polynomials (in which case it is also easy to show $\boldsymbol{\alpha} \in \mathcal{C}$ ). However, for our analysis in $\S 5$, we need a theorem applicable when $c_{n}$ is quadratic in $n$.

THEOREM 4.2. Let

$$
c_{n}=O\left(n^{2}\right), \quad \alpha_{n}=\frac{1}{4}+\left(1+r_{n}\right) /\left[16 n^{2}\right] .
$$

Then if $r_{n} \rightarrow 0, \sigma=0$. Moreover (i) if $r_{n} \leqslant s_{n}$ where $s_{n} \geqslant 0$ and either $s_{n}=O\left(n^{-1}\right)$ or $\sum s_{n}$ converges, then $\psi \in \mathbb{Q}(0)$; (ii) if $r_{n} \geqslant 0$ and $\sum n^{-1} r_{n}=\infty$, then $\psi \notin \mathbb{Q}(0)$.

Proof. Choose $\beta_{n}$ as in the proof of Theorem 4.1. Then $r_{n} \rightarrow 0$ implies $A_{n}(\boldsymbol{\beta}) \rightarrow 0$. As before we can choose $s_{n}$ so that either $\boldsymbol{\beta} \in \mathcal{E}$ or $\boldsymbol{\beta} \notin \mathcal{E}$ and conclude $\sigma=0$. But according to Theorem 2.2, the conditions in (i) yield $\alpha \in \mathcal{E}$ while those in (ii) say that $\boldsymbol{\alpha} \notin \varepsilon$. Theorem 1.1(iii) then yields the desired conclusions.

We next obtain a theorem which is, in a sense, an analog of a theorem of Geronimo and Case [11] for the bounded case. (For the proof of continuity at $\sigma$, see Theorem 4.5.)

THEOREM 4.3. Let

$$
\sum c_{n}^{-1}=\infty \quad \text { and } \quad \sum n\left|\alpha_{n}-\frac{1}{4}\right|<\infty
$$

Then $\psi \in \mathbb{Q}(0)$. 
Proof. The condition $\Sigma n\left|\alpha_{n}-\frac{1}{4}\right|<\infty$ implies $\alpha \in \mathcal{E}$ [10, Lemma 2] so $\sigma \geqslant 0$. Referring to (1.5), we see that for each $x>0$ there is an $N=N(x)$ such that $\varepsilon_{n}(x) \geqslant 0, \sum \varepsilon_{n}(x)=\infty(n \geqslant N)$. Thus $\alpha(x) \notin \mathcal{E}[8$, p. 99] for all $x>0$; hence $\boldsymbol{\sigma}=0$. Since $\boldsymbol{\alpha}(0) \in \mathcal{E}, \psi \in \mathbb{Q}(0)$.

We next take up the question of continuity of $\psi$ at the spectral limit point $\sigma$. We will need to recall some identities for the orthonormal polynomials corresponding to (0.1):

$$
p_{n}(x)=\left(\lambda_{1} \lambda_{2} \cdots \lambda_{n+1}\right)^{-1 / 2} P_{n}(x) \quad\left(\lambda_{1}=\psi(\infty)-\psi(-\infty)\right) .
$$

If $c_{n}^{(N)}>0$ and $\boldsymbol{\alpha}^{(N)}$ is a chain sequence, it has a parameter sequence $\left\{g_{n}\right\}$ given by $[9$, p. 362]

$$
g_{n}=1+\frac{P_{n+N+1}(0)}{c_{n+N+1} P_{n+N}(0)}, \quad n \geqslant 0 .
$$

Note that if $N=0, g_{0}=0$ so these become minimal parameters. From (4.1) we get $[9$, loc. cit.]

$$
\begin{gathered}
{\left[\frac{p_{n+N+1}(0)}{p_{n+N}(0)}\right]^{2}=\left[1+\frac{1-g_{n}-g_{n+1}}{g_{n+1}}\right]\left[1+\frac{c_{n+N+1}-c_{n+N+2}}{c_{n+N+2}}\right],} \\
p_{n+N}^{2}(0)=\frac{c_{N} p_{N}^{2}(0)}{c_{n+N+1}} \prod_{k=1}^{n} \frac{1-g_{k-1}}{g_{k}} .
\end{gathered}
$$

We cite the classical result that if the Hamburger moment problem is determined, then the jump of $\psi$ at $x$ is

$$
\rho(x)=\left[\sum_{n=0}^{\infty} p_{n}^{2}(0)\right]^{-1}
$$

(see [16, Corollary 2.6]).

Theorem 4.4. Let $\boldsymbol{\alpha}^{(N)} \in \mathcal{C}, \alpha_{n}^{(N)} \geqslant \frac{1}{4}, \sigma=0$.

(i) If $\sum c_{n}^{-1}=\infty, \psi$ is continuous at 0 .

(ii) If $\sum n^{2} c_{n}^{-1}<\infty$, $\psi$ has a positive jump at 0 .

Proof. From $\boldsymbol{\alpha}_{n}^{(N)} \geqslant \frac{1}{4}$ follows

$$
n /[2(n+1)] \leqslant g_{n} \leqslant \frac{1}{2}, \quad n \geqslant 1 .
$$

Thus (4.3) yields

$$
c_{N+1} p_{N}^{2}(0) c_{n+N+1}^{-1} \leqslant p_{n+N}^{2}(0) \leqslant c_{N+1} p_{N}^{2}(0)(n+1)(n+2) c_{n+N+1}^{-1} .
$$

The theorem thus follows from (4.4).

Theorem 4.5. Let $\sum c_{n}^{-1}=\infty, \sum n\left|\alpha_{n}-\frac{1}{4}\right|<\infty$. Then $(\psi \in \mathbb{Q}(0)$ and $) \psi$ is continuous at 0 .

Proof. Choose $N$ so that

$$
\sum n\left|\varepsilon_{n}^{(N)}\right| \leqslant \frac{1}{4} \quad\left(\alpha_{n}=\frac{1}{4}+\varepsilon_{n}\right) .
$$


By [10, Lemma 2], $\boldsymbol{\alpha}^{(N)} \in \mathcal{C}$ and for its parameters (4.1) we have $g_{n} \leqslant \frac{1}{2}+2 G_{n}$ where $G_{n}=\sum_{\nu=1}^{\infty}\left|\varepsilon_{n+\nu}^{(N)}\right|$. Thus

$$
\frac{1-g_{n-1}}{g_{n}} \geqslant \frac{1-4 G_{n-1}}{1-4 G_{n}}>0 \text { for } n \geqslant 2
$$

Thus

$$
A_{n} \equiv \prod_{k=1}^{n} \frac{1-g_{k-1}}{g_{k}} \geqslant \frac{1-g_{0}}{g_{1}} \prod_{k=2}^{n}\left[1-\frac{4\left(G_{k-1}+G_{k}\right)}{1+4 G_{k}}\right]>0 .
$$

But

$$
\sum_{n=2}^{\infty}\left(G_{n-1}+G_{n}\right) \leqslant 2 \sum_{n=0}^{\infty} G_{n}-\left|\varepsilon_{1}^{(N)}\right|=2 \sum_{n=1}^{\infty} n\left|\varepsilon_{n}^{(N)}\right|-\left|\varepsilon_{1}^{(N)}\right| .
$$

It follows that $A_{n}$ is, for all $n$, bounded below by a positive number. Reference to (4.3) thus shows that the divergence of $\sum c_{n}^{-1}$ implies that of $\sum p_{n}^{2}(0)$.

The next theorem and proof is a rather obvious adaptation of our earlier work for the bounded case [9, Theorem 6].

THEOREM 4.6. Let $c_{n}^{(N)}>0, \boldsymbol{\alpha}^{(N)} \in$ e. If

$$
\begin{gathered}
\alpha_{n}^{(N)} \geqslant \frac{1}{4}-\frac{a}{16 n(n+1)}, \quad 0<a<3, \\
\liminf _{n \rightarrow \infty} n\left(1-\frac{c_{n}}{c_{n+1}}\right)>\sqrt{a+1}-2
\end{gathered}
$$

then $(\sigma \geqslant 0$ and $) \psi$ is continuous at 0 .

Proof. We can rewrite (4.2) as

$$
\left[\frac{p_{n+N}(0)}{p_{n+N+1}(0)}\right]^{2}-1=\frac{1-g_{n-1}-g_{n}}{g_{n}}+\left(\frac{1-g_{n-1}}{g_{n}}\right)\left(1-\frac{c_{n+N}}{c_{n+N+1}}\right) .
$$

Using inequalities derived from (4.6) in the proof of [9, Theorem 6], we find

$$
\frac{n\left(1-g_{n-1}-g_{n}\right)}{g_{n}} \geqslant-\frac{s n(2 n+3)}{(n+1)(n+s+2)}>-2 s
$$

where $2 s=\sqrt{1+a}-1<1$. Since $\alpha \in \mathcal{E}$, (4.6) implies $\alpha_{n} \rightarrow \frac{1}{4}$ which says that $g_{n} \rightarrow \frac{1}{2}$. Using (4.7) and (4.8), we conclude

$$
\liminf _{n \rightarrow \infty} n\left\{\left[\frac{p_{n+N}(0)}{p_{n+N+1}(0)}\right]^{2}-1\right\}>-1 .
$$

The divergence of $\Sigma p_{n}^{2}(0)$ now follows from Raabe's test.

Theorem 4.6 is applicable to the Laguerre polynomials $L_{n}^{(c)}(x)$ if (but only if) $-1<c<2$. The difficulty we encounter in our attempts to extend the theorem lies in the fact that we do not know if $\left\{g_{n}\right\}$ in (4.1) is the maximal parameter sequence for $\boldsymbol{\alpha}^{(N)}$. Thus we are forced to compare $g_{n}$ with the maximal parameters of the chain sequence used on the right side of (4.6). Now all nonmaximal parameter sequences of a chain sequence are asymptotically equal $[8, p .102]$. Therefore, if we 
knew $\left\{g_{n}\right\}$ was not maximal, we could use a smaller chain sequence on the right side of (4.6) and compare $g_{n}$ with its minimal parameters to get an inequality such as (4.8).

One way to conclude that the $g_{n}$ are themselves actually minimal is to impose conditions such that $\boldsymbol{\alpha}$ is a chain sequence. We do this in the next theorem which is then applicable to $\left\{L_{n}^{(c)}(x)\right\}$ for all $c>-1$. The price we pay to achieve this is the fact that this theorem will not apply if there are any spectral points smaller than 0 .

THEOREM 4.7. Let $c_{n}>0(n \geqslant 1)$ and

$$
\begin{gathered}
\liminf _{n \rightarrow \infty} n\left(1-\frac{c_{n}}{c_{n+1}}\right)>-2, \\
\alpha_{n} \leqslant \frac{1}{4}+\left(4\left(4 n^{2}-1\right)\right)^{-1}, \quad n \geqslant 1 .
\end{gathered}
$$

Then $\psi$ is continuous at 0 .

Proof. We have the identity

$$
\beta_{n} \equiv \frac{1}{4}+\frac{1}{4\left(4 n^{2}-1\right)}=\left(1-\frac{n-1}{2 n-1}\right) \frac{n}{2 n+1} .
$$

Thus $\boldsymbol{\beta}$ is a chain sequence with minimal parameters $n(2 n+1)^{-1}(n \geqslant 0)$. (These are also maximal parameters.) By Theorem 1.2 and (4.10), $\alpha \in \mathcal{C}$ and, since $c_{n}>0$, the $g_{n}$ in (4.2) are its minimal parameters. Also, $g_{n} \leqslant n(2 n+1)^{-1}$; hence

$$
\left(1-g_{n-1}-g_{n}\right) / g_{n} \geqslant(2 n-1)^{-1} \text {. }
$$

The latter together with (4.11) now yields exactly as in the proof of Theorem 4.6

$$
\liminf _{n \rightarrow \infty} n\left\{\left[\frac{p_{n+N}(0)}{p_{n+N+1}(0)}\right]^{2}-1\right\}>-1 .
$$

Once again, Raabe's test gives the desired conclusion.

We note that under the conditions of Theorem 4.7, we also have $\sigma(\mathbf{P}) \geqslant \xi_{1}(\mathbf{P}) \geqslant 0$. If we assume further that $\alpha_{n}=\frac{1}{4}+O\left(n^{-2}\right)$, we can then conclude additionally that the spectral interval is precisely $[0, \infty), \psi \in \mathcal{Q}(0)$, and the zeros are dense in $[0, \infty)$.

5. Examples. In the majority of examples of specific systems of orthogonal polynomials which have appeared in the literature and which have $c_{n} \rightarrow \infty$, both $c_{n}$ and $\lambda_{n}$ are polynomials in $n$ or both are asymptotically exponential functions of $n$. In the latter cases, $\left\{\alpha_{n}\right\}$ always converges so $|\sigma|=\infty$ (by (3.1) and Theorem 3.2).

We will here consider the polynomial cases. Actually our work will apply to nonpolynomial coefficients having "polynomial-like" asymptotic forms (see (5.1)). We will cite one recent example with coefficients which are rational functions of $n$ to which our theorems apply.

In view of (3.1), it is sufficient to consider

$$
\begin{aligned}
c_{n+1} & =2 n^{k}+a n^{k-1}+b n^{k-2}+R_{n}, \\
\lambda_{n+1} & =n^{2 k}+f n^{2 k-1}+g n^{2 k-2}+S_{n},
\end{aligned}
$$


where $R_{n}=o\left(n^{k-2}\right), S_{n}=o\left(n^{2 k-2}\right)$, and $f, b$ and $S_{n}$ must be restricted so that $\lambda_{n}>0$.

We have

$$
\alpha_{n}=\frac{1}{4}+\frac{(f-a+k) n^{2 k-1}+(g-G) n^{2 k-2}+O\left(n^{2 k-3}\right)}{4\left[n^{2 k}+(a-k) n^{2 k-1}+G n^{2 k-2}+O\left(n^{2 k-3}\right)\right]}
$$

where $G=b+\frac{1}{4}(a-2 k+1)^{2}-\frac{1}{4}\left(2 k^{2}-2 k+1\right)$. Note that we can obtain $\alpha_{n}(x)$ from (5.2) by replacing the constant term, say $c$, in $c_{n}$ by $c-x$.

THEOREM 5.1. When $k=1$, $\sigma=a-f-1$

$\psi \in \mathbb{Q}(\sigma)$ if and only if $f^{2} \geqslant 4(g-b)$;

$\psi$ is continuous at $\sigma$ if $f^{2}<4(g-b+1)$.

In the special case $b=R_{n}=S_{n}=0, \psi$ is continuous at $\sigma$ for all permissible values of $f$ and $g$.

Proof. When $k=1, a$ is the constant term so we replace it by $a-x$ in (5.2). It is then easy to see that there exists an $N$ such that

$$
\begin{array}{cc}
\alpha_{n}(x)<\frac{1}{4} \quad(n \geqslant N) \quad \text { if } f-a+1+x<0 ; \\
\alpha_{n}(x)>\frac{1}{4} \quad(n \geqslant N) \quad \text { and } \quad \sum\left[\alpha_{n}(x)-\frac{1}{4}\right]=\infty
\end{array}
$$

if $f-a+1+x>0$.

It follows that $\boldsymbol{\alpha}(x) \in \mathcal{E}$ if $x<a-f-1$ and $\boldsymbol{\alpha}(x) \notin \mathcal{E}$ if $x>a-f-1$. That is, $\sigma=a-f-1$.

We then have

$$
\alpha_{n}(\sigma)=\frac{1}{4}+\frac{1+4 g-4 b-f^{2}+O\left(n^{-1}\right)}{16\left[n^{2}+f n+O(1)\right]} .
$$

According to Theorem 2.2, $\boldsymbol{\alpha}(\sigma) \in \mathcal{E}$ if and only if $4 g-4 b-f^{2} \leqslant 0$.

As for continuity at $\sigma$, Theorem 4.6 shows that $\psi$ is continuous at $\sigma$ if $f^{2}<4 g+4$ $-4 b$. Unfortunately, Theorem 4.7 is difficult to apply in the general case since a comparison must be made for $n \geqslant 1$ and this will involve the asymptotic order terms $R_{n}$ and $S_{n}$. We therefore specialize to the polynomial case $b=R_{n}=S_{n}=0$. Theorem 4.7 shows that in this case $\psi$ is continuous at $\sigma$ if either $f \geqslant 0$ and $f^{2} \geqslant 4 g$ or $-1<f<0$ and $f^{2}+f \geqslant 3 g$. This leaves open only the pairs $(f, g)$ for which $f \leqslant-1$ and $f^{2} \geqslant 4 g+1$. However, for any such pair, there will be at least one $m \geqslant 2$ for which $\lambda_{m} \leqslant 0$.

Turning next to $k \geqslant 2$, we note that $\alpha_{n}(x)$ is given by $\alpha_{n}$ in (5.2) where

(i) if $k=2, b$ is replaced by $b-x$, and $a, f$ and $g$ are independent of $x$;

(ii) if $k>2, a, b, f$ and $g$ are independent of $x$.

THEOREM 5.2. (1) When $k=2$,

(i) if $f<a-2, \sigma=\infty$;

(ii) if $f>a-2, \sigma=-\infty$;

(iii) if $f=a-2, \sigma=(a-3)^{2} / 4+b-g-1, \psi \in \mathbb{Q}(\sigma)$ and $\psi$ is continuous at $\sigma$. 
(2) When $k \geqslant 3, \sigma=\infty$ if either $f<a-k$, or $f=a-k$ and

$$
g \leqslant(a-2 k+1)^{2} / 4+b-k(k-1) / 2 .
$$

In all other cases, $\sigma=-\infty$.

Proof. We first observe that if $f<a-k$, then $\alpha_{n}(x)<\frac{1}{4}$ for all sufficiently large $n$, hence $\boldsymbol{\alpha}(x) \in \mathcal{E}$ for all $x$ so $\sigma=\infty$. But if $f>a-k$, then $\alpha_{n}(x)>\frac{1}{4}$ for sufficiently large $n$ and $\alpha_{n}(x)=\frac{1}{4}+O\left(n^{-1}\right)$. That is, $\boldsymbol{\alpha}(x) \notin \mathcal{E}$ so $\sigma=-\infty$.

Taking $f=a-k$, we rewrite (5.2) as

$$
\alpha_{n}(x)=\frac{1}{4}+\left(1+e_{n}(x)\right) /\left[16 n^{2}\right]
$$

where $e_{n}(x)=4(g-G)-1+O\left(n^{-1}\right)$. Reference to Theorem 2.2 shows that $\alpha(x)$ $\in \mathcal{E}$ if and only if $4(g-G) \leqslant 1$. In particular, for $k=2$,

$$
G=(a-3)^{2} / 4+b-1-x,
$$

so this yields

$$
\sigma=\frac{1}{4}(a-3)^{2}+b-g-1
$$

and $\psi \in \mathscr{Q}(\sigma)$. Theorem 4.6 shows $\psi$ is continuous at $\sigma$.

On the other hand, $4(g-G)$ is independent of $x$ for $k>2$. It follows that $\boldsymbol{\alpha}(x) \in \mathcal{E}$ if and only if $4(g-G) \leqslant 1$ independently of $x$. That is, if

$$
g \leqslant \frac{1}{4}(a-2 k+1)^{2}+b-\frac{1}{2} k(k-1),
$$

then $\sigma=\infty$ while $\sigma=-\infty$ otherwise.

While there are many well-known examples in which $c_{n}$ is linear and $\lambda_{n}$ is quadratic, there does not seem to be any in the literature with $c_{n}$ cubic or higher degree. The only examples we have seen in which $c_{n}$ is quadratic and $\alpha_{n} \rightarrow \frac{1}{4}$ are a pair of sequences, related to Meixner polynomials of the second kind, which were encountered by Al-Salam [1]. The first of these (the second is very similar) is given by the recurrence

$$
\begin{aligned}
U_{n+1}(x)= & {\left[x-8 n^{2}-(4 n+1) h\right] U_{n}(x) } \\
& -2 n(2 n-1)(2 n+h-2)(2 n+h-1) U_{n-1}(x)
\end{aligned}
$$

(see also [8, pp. 180-181]). Renormalizing by setting $P_{n}(x)=4^{-n} U_{n}(4 x)$, we obtain for $(0.1)$,

$$
\begin{gathered}
c_{n+1}=2 n^{2}+h n+h / 4, \\
\lambda_{n+1}=n^{4}+(h-2) n^{3}+\left(h^{3}-5 h+5\right) n^{2} / 4+O(n) .
\end{gathered}
$$

Thus, referring to (5.1), $f=a-2$ so Theorem 2 yields $\sigma=0$ and other conclusions consistent with the known properties of these polynomials.

Askey and Wilson [3] have recently discussed the symmetric polynomials $S_{n}(x)=$ $S_{n}(x ; \alpha, \gamma)$ satisfying

$$
\begin{gathered}
x S_{n}(x)=A_{n} S_{n+1}(x)+C_{n} S_{n-1}(x), \\
A_{n}=\frac{(n+2 \gamma)(n+2 \alpha+2 \gamma-1)}{2(2 n+2 \alpha+2 \gamma-1)}, \quad C_{n}=\frac{n(n+2 \alpha-1)}{2(2 n+2 \alpha+2 \gamma-1)} .
\end{gathered}
$$


They show that the polynomials $T_{n}(x)=S_{2 n}\left(x^{1 / 2}\right)$ are special cases of a class of hypergeometric polynomials whose orthogonality relations were discovered by Wilson [18]. We consider the corresponding monic polynomials and renormalize:

$$
P_{n}(x)=4^{n} A_{0} A_{1} \cdots A_{n-1} S_{2 n}\left(x^{1 / 2} / 4\right) .
$$

For $\left\{P_{n}(x)\right\}$ we then obtain $(0.1)$ with

$$
\begin{gathered}
c_{n+1}=2 n^{2}+(2 \alpha+2 \gamma-1) n+2^{-1}(\alpha+\gamma)+A+O\left(n^{-2}\right), \\
\lambda_{n+1}=n^{4}+(2 \alpha+2 \gamma-3) n^{3}+\left[2^{-1}(\alpha+\gamma-2)(2 \alpha+2 \gamma-3)+A\right] n^{2}+O(n),
\end{gathered}
$$

where $A=-\frac{1}{8}-(\alpha-\gamma)^{2} / 2$ and the 0 terms are rational in $n$. Thus $c_{n}$ and $\lambda_{n}$ are rational functions of $n$ which satisfy (5.1). Once again, Theorem 5.2 yields $\sigma=0$ and other conclusions consistent with known facts.

As a final example, consider $A_{n}(x)=A_{n}(x ; c)$ defined by [8, p. 193]

$$
\begin{aligned}
A_{n+1}(x)=\left[x-(2 n+1)^{2} a\right] A_{n}(x)-4 n^{2}\left(4 n^{2}-1\right) c A_{n-1}(x), \\
0<c<1, a=1+c .
\end{aligned}
$$

These polynomials have been studied by Stieltjes and by Carlitz. They are orthogonal with respect to a discrete distribution whose spectral points are proportional to the odd integers. We note that we have $\alpha_{n} \rightarrow c(1+c)^{-2}<\frac{1}{4}$ for $c \neq 1, c>0$, and that $A_{n}\left(x ; c^{-1}\right)=c^{-n} A_{n}(c x ; c)$ so that orthogonality relations are known for $c>1$ also. This suggests looking into the case $c=1$. Putting $P_{n}(x)=4^{-n} A_{n}(4 x ; 1)$, we find

$$
c_{n+1}=2 n^{2}+2 n+\frac{1}{2}, \quad \lambda_{n+1}=n^{4}-n^{2} / 4 .
$$

Theorem 5.2 then shows that $\sigma=0, \psi$ is continuous at 0 and has at most finitely many spectral points smaller than 0 . (It is easy to show there are actually no spectral points smaller than 0 .) Since the zeros will be dense in $(0, \infty)$, this suggests looking for a weight function on $(0, \infty)$.

Richard Askey has informed the author that these polynomials can in fact be identified with the orthogonal polynomials

$$
D_{n}\left(x^{2} ; a, b, c\right)={ }_{3} F_{2}\left(\begin{array}{cc}
-n, a+i x, a-i x & \\
a+b, a+c
\end{array}\right)
$$

which are the limiting cases, $d \rightarrow \infty$, of the polynomials

$$
W_{n}\left(x^{2} ; a, b, c, d\right)={ }_{4} F_{3}\left(\begin{array}{cc}
-n, n+a+b+c+d-1, a+i x, a-i x & ; 1 \\
a+b, a+c, a+d
\end{array}\right)
$$

discussed in [18]. The three term recurrence formula for the latter was found by Wilson in his thesis [19]. This part of his thesis has not yet been published, but the formula can also be found from the more general formula in [20] by letting $q \rightarrow 1$. For the special case (5.4), comparison of the resulting recurrence formula with (5.3) then shows that

$$
A_{n}(x ; 1)=(-1)^{n}(2 n) ! D_{n}\left(x / 4 ; 0,1, \frac{1}{2}\right) .
$$


Wilson's orthogonality relation $[\mathbf{1 8},(4.4)]$ then yields

$$
\int_{0}^{\infty} A_{m}(x ; 1) A_{n}(x ; 1) \operatorname{csch} \frac{\pi x^{1 / 2}}{2} d x=0 \quad(m \neq n) .
$$

\section{REFERENCES}

1. W. A. Al-Salam, Characterization of a certain class of orthogonal polynomials related to elliptic functions, Ann. Mat. Pura Appl. 67 (1965), 75-94.

2. W. A. Al-Salam and L. Carlitz, Some orthogonal q-polynomials, Math. Nachr. 30 (1965), 47-61.

3. R. Askey and J. Wilson, A set of hypergeometric orthogonal polynomials, SIAM J. Math. Anal. (to appear).

4. O. Blumenthal, Über die entwicklung einer willkürlichen Funktion nach den Nennern des Kettenbruches für $\int_{-\infty}^{\infty}[\phi(\xi) /(z-\xi)] d \xi$, Dissertation, Göttingen, 1898.

5. T. S. Chihara, Chain sequences and orthogonal polynomials, Trans. Amer. Math. Soc. 104 (1962), $1-16$.

6. On recursively defined orthogonal polynomials, Proc. Amer. Math. Soc. 16 (1965), 702-710.

7. __ On indeterminate Hamburger moment problems, Pacific J. Math. 27 (1968), 475-484.

8. __ An introduction to orthogonal polynomials, Gordon and Breach, New York, 1978.

9. Orthogonal polynomials whose distribution functions have finite point spectra, SIAM J. Math. Anal. 11 (1980), 358-364.

10. T. S. Chihara and P. G. Nevai, Orthogonal polynomials and measures with finitely many point masses (to appear).

11. J. S. Geronimo and K. M. Case, Scattering theory and polynomials orthogonal on the real line, Trans. Amer. Math. Soc. 258 (1980), 467-494.

12. P. G. Nevai, Orthogonal polynomials, Mem. Amer. Math. Soc., no. 213 (1979).

13. __ Distribution of zeros of orthogonal polynomials, Trans. Amer. Math. Soc. 249 (1979), $341-361$.

14. Orthogonal polynomials defined by a recurrence relation, Trans. Amer. Math. Soc. 250 (1980), 369-384.

15. J. Sherman, On the numerators of the convergents of the Stieltjes continued fractions, 'Trans. Amer. Math. Soc. 35 (1933), 64-87.

16. J. Shohat and J. D. Tamarkin, The problem of moments, Math. Surveys No. 1, Amer. Math. Soc., Providence, R. I., 1943, 1950.

17. H. S. Wall, Analytic theory of continued fractions, Van Nostrand, Princeton, N.J., 1948.

18. J. Wilson, Some hypergeometric orthogonal polynomials, SIAM J. Math. Anal. 11 (1980), 690-701.

19. Hypergeometric series recurrence relations and some new orthogonal functions, Ph.D. Thesis, Univ. of Wisconsin, Madison, 1978.

20. R. Askey and J. Wilson, A set of orthogonal polynomials that generalize the Racah coefficients or 6-j symbols, SIAM J. Math. Anal. 10 (1979), 1008-1016.

Department of Mathematical Sciences, Purdue University Calumet, Hammond, Indiana 46323 